\title{
EL ESTATUTO DEL ESTUDIANTE UNIVERSITARIO EN ESPAÑA: DERECHOS Y RESPONSABILIDADES
}

\author{
THE STATUS OF COLLEGE STUDENT IN SPAIN: \\ RIGHTS AND RESPONSIBILITIES
}

Recibido: 07/04/2014

Revisado: 06/05/2015

Aceptado: 29/05/2015

Ignacio Jiménez Soto ${ }^{1}$

\section{RESUMEN}

Según la disposición adicional decimocuarta de la Ley Orgánica de Modificación a la Ley de Universidades (LOMLOU) Ley 4/2007, de 12 de abril, se establece un plazo de un año para la elaboración del Estatuto del Estudiante Universitario, con algo de retraso, el día 30 de diciembre de 2010, el Consejo de Ministros aprobó el Real Decreto 1791/2010, por el que se aprueba el Estatuto del Estudiante Universitario (BOE de 31 de diciembre de 2010). Es pues, el texto aprobado por el Real Decreto, el que da origen a esta ponencia, que tratará sobre aquellos aspectos que pueden ser más relevantes una vez que, han transcurrido cuatro años desde la entrada en vigor de una norma muy relevante en el sistema universitario español, teniendo en cuenta el conjunto de derechos y obligaciones que contiene.

\section{ABSTRACT}

According to the fourteenth additional provision of the Law on Amendments to the Universities Act (LOMLOU) Law 4/2007 of 12 April, a period of one year is set for the preparation of the Statute of the University Student, with some delay, on December 30, 2010, the Council of Ministers approved Royal Decree 1791/2010, approving the University Student Statute (BOE of December 31, 2010) is approved. It is therefore the text approved by Royal Decree, which gives rise to this paper, which will focus on those aspects that may be more relevant once four years have passed since the entry into force of a relevant standard in the system Spanish university, taking into account all rights and obligations contained therein.

\section{DE LA CALMA CHICHAA LOS FUERTES VIENTOS}

El barco del Estatuto del Estudiante Universitario, ha tenido una larga travesía de más de tres años y medio, de navegación, desde que salió del puerto de la LOMLOU en abril de 2007, hasta que ha llegado a su destino en el Boletín Oficial del Estado (31 de diciembre de 2010). En su cuaderno de navegación, encontrado en un camarote de proa, se puede leer cierta crítica, escrita por algún oficial de la embarcación, donde se queja de las dificultades que han tenido en la travesía por la falta de vientos favorables; así como por el hermetismo con el que, en algún momento, los armadores del barco han mantenido la travesía. De ahí que, algún plumilla, avezado, haya escrito en cierto rotativo: que da la impresión de que lo importante es que el barco llegue a buen puerto, y que no se comente la travesía.

Y es que símil a parte, la elaboración del Estatuto del Estudiante Universitario, norma muy importante como así lo han manifestado las autoridades académicas (Ministerio, Rectores, etc.,), se asemeja a la travesía de un barco, de vela, en alta mar, con paradas continuas y travesías cortas, como demuestra el tiempo transcurrido desde su nacimiento por la propia Ley.

\section{DE LAS SOMBRAS A LAS LUCES}

No hay texto jurídico, independientemente del procedimiento realizado en su elaboración, que no presente "sombras" y "luces", aciertos o desaciertos; y que, en definitiva, su exposición no tiene más finalidad que mejorar la norma a través de la crítica sana y constructiva, que es lo que pretendemos a través de nuestros trabajos. Ya, en su momento, manifestamos el acierto del MEC por haber elaborado un documento (borrador) sobre el Estatuto, y que incluso, la existencia del mismo, podía ser el primer éxito de la batalla que le quedaba al Estatuto por ganar.

\subsection{El omnipresente Espacio Europeo de Educación Superior}

Comenzamos nuestra intervención con la Declaración de Bolonia de 19 de junio de 1999, a la que algunos autores como Martín Rebollo no habían dudado en calificarla de auténtico mito; sin embargo, y aún aceptando tal calificación, lo cierto es que al amparo de ésta va a gravitar buena parte del sistema universitario, donde nosotros justificamos la necesidad del Estatuto, sobre todo, en el hecho de cohesionar todo el elenco de derechos y obligaciones de los estudiantes, respetando, por supuesto, la autonomía universitaria, y sin que esto suponga mermar el Espacio Europeo de Educación Superior (EEES). Por lo tanto, podríamos preguntarnos ¿qué importancia ha tenido el EEES en la elaboración del Estatuto del Estudiante Universitario?, máxime cuando la necesidad del mismo es evidente ante la ingente cantidad de materias que requieren una normativa de acuerdo con los nuevos tiempos: regulación de la valoración del conocimiento, el arcaico régimen disciplinario, la permanencia en las universidades, la tan deseada calidad en la Universidad española, etc., la respuesta a ello podemos encontrarla, en el impulso que ha supuesto la Declaración de Bolonia, para activar el aparato normativo en el ámbito universitario, que estaba dormitando en la más absoluta de las indeferencias, como demuestra el escaso desarrollo normativo, en lo que afecta al régimen de derechos y deberes en los universitarios, pese al esfuerzo y modernización que supuso la promulgación de la Ley Orgánica 11/1983, de 25 de agosto, Reforma Universitaria (LRU), y que obligó a un cambio profundo en el sistema universitario, que a su vez generó un compendio de normativas universitarias de marcado carácter disperso, y poco cohesionadas entre éstas, como se puede ver, a título de ejemplo, en el auténtico "reino de Taifas" en que se convirtió la compensación de créditos, o el escaso interés por determinar el régimen de permanencia en las Universidades españolas.

En definitiva, el EEES, no solamente fomenta la creación del Estatuto, sino que constituye el pretexto perfecto, 
para dar respuesta a la creación de un nuevo régimen jurídico que demandan, principalmente, los estudiantes, aunque sus efectos se extiendan a toda la comunidad universitaria.

\subsection{Algunas reflexiones y consideraciones sobre el Estatuto}

Es importante destacar desde el punto de vista sociojurídico, la perspectiva con la que nace el Estatuto, en un momento en que tomados los datos del curso 2008/2009, el Sistema Universitario Español lo conformaron 1.504.276 estudiantes: 1.358.875 de primer y segundo ciclo, 18.353 de grado (enseñanzas adaptadas al EEES), 49.799 de master oficiales y 77.249 de doctorado, de los que 5.987 cursaron estudios adaptados al a las directrices del EEES. Con estos datos, se pretende aumentar la implicación de los estudiantes en la vida universitaria, desde el reconocimiento de sus derechos, hasta el marco legal que rige la convivencia en la Universidad, para finalizar con la creación del órgano consultivo más importante de representación estudiantil.

Así pues, el Estatuto va a nacer con la rémora de una década en la que se ha producido un descenso de estudiantes universitarios, sobre todo por el descenso de la población de edades entre 18 y 24 años, descenso que ha alcanzado el $18.3 \%$, situando la tasa neta de escolarización en ese tramo en el $23.8 \%$, y que desde el punto de vista positivo se espera en los próximos años el incremento del número de estudiantes universitarios, donde la creación de las estructuras, como puede ser el Consejo de Estudiantes Universitario del Estado, que presenta la norma puede fortalecer el papel de los estudiantes dentro del sistema universitario español.

El tiempo transcurrido, cuatro años, ha permitido ver como al rebufo de la norma las Universidades se han dedicado a producir numerosas normativas de carácter reglamentarios, sobre todo, para adaptar sus normas internas el Estatuto del Estudiante Universitario: de naturaleza académica y sobre los órganos de participación de los estudiantes; igualmente, se siente en la comunidad universitaria la frustración de no poder contar con una Ley sobre Disciplina Académica que derogue el vetusto y obsoleto Decreto de 1954, en pleno régimen franquista, y declarado vigente en su mayor parte por nuestro Tribunal Supremo; se aprecia un notable desconocimiento de la norma tanto en los profesores como en los propios alumnos, quizás por desinterés interesado, o por la propia apatía universitaria y por último el Estatuto ha sido un instrumento muy valioso para los Defensores Universitarios al tener una norma de indudable referencia para ejercitar su magistratura en defensa de los derechos de los estudiantes, así como en el cumplimiento de sus deberes.

\subsection{Sobre el Contenido}

Del contenido del Real Decreto, podemos decir, sintéticamente, que consta de un preámbulo, una parte dispositiva (artículo único) donde se aprueba el Estatuto del Estudiante Universitario, una disposición adicional primera en lo que afecta a la cobertura de seguro, y donde el Gobierno estudiará las contingencias actuales del seguro escolar; seguida de una disposición adicional segunda, por la que el Gobierno presentará un proyecto de ley reguladora de la potestad disciplinaria, y dos disposiciones finales: la primera, en la que se expresa el título competencia, como norma básica, a excepción del Capítulo XI del Consejo del Estudiante Universitario, y la segunda que atañe al Reglamento del Consejo del Estudiante Universitario, que se elaborará en el plazo de seis meses desde su constitución.

Centrándonos en su contenido, el texto presenta 67 artículos agrupados en dieciséis capítulos y cuyo contenido resumido es el siguiente:

En el Capítulo I se establece el objeto y el ámbito de aplicación de la norma a todos los estudiantes de las universidades públicas y privadas españolas.

En el Capítulo II se regulan los derechos y deberes de los estudiantes, distinguiendo entre los Derechos generales:
Formación académica de calidad, Orientación, Reconocimiento de la participación, Movilidad, Becas y ayudas, etc., y derechos particulares según su condición de estudiante de Grado, Master, Doctorado, Formación Continua y Extensión Universitaria.

En el capítulo III nos encontramos el acceso y la admisión a la Universidad con remisión a la legislación vigente, y con especial consideración a los estudiantes con discapacidad.

Por su parte en el Capítulo IV se regula la movilidad estudiantil y su reconocimiento, en cualquiera de los tres ciclos de enseñanza, donde se implica a las Universidades a que realicen programas específicos de movilidad nacional e internacional.

Es en el Capítulo $V$ donde podemos encontrar las actividades de orientación al estudiante, y la configuración de sistemas tutoriales, desarrollados por el profesorado y el personal especializado, distinguiendo entre la tutoría de carrera y la tutoría de materia o asignatura.

La programación docente del estudiante de enseñanzas que conducen a la obtención de un título oficial, con lo que suponen las prácticas externas, el derecho a una evaluación objetiva, la comunicación de las calificaciones y la revisión, así como el reconocimiento de determinadas actividades culturales, deportivas, de representación, solidarias y de cooperación, lo encontramos en el Capítulo VI.

Por su parte si queremos encontrar la regulación docente de los estudiantes que cursan enseñanzas no conducentes a un título oficial, es el Capítulo VII quien lo determina.

La representación y participación estudiantil, con todo lo que suponen los derechos y responsabilidades de los representantes estudiantiles, lo podemos encontrar en el Capítulo VIII.

Los principios básicos de la política de becas y ayudas al estudio, sentados en la equidad y eficacia en la gestión de la política de becas, se acomodan en el Capítulo IX.

En el Capítulo $X$ se recoge el fomento de la convivencia y la corresponsabilidad en la Universidad. Para ello, se prevé la posibilidad de la creación de comisiones de ética y responsabilidad en los centros, como cauce de análisis y propuestas y sin carácter sancionador, integrando a los Defensores Universitarios como promotores de la cultura de la ética y la convivencia.

La regulación del Consejo de Estudiantes Universitario del Estado, como órgano de deliberación, consulta y participación de los estudiantes universitarios, se halla detenidamente regulado: estructura, composición, competencias, etc., en el capítulo XI.

El Capítulo XII nos encontramos la actividad deportiva de los estudiantes, considerada como un componente de la formación integral del alumno, y donde las Universidades promoverán la actividad deportiva, los hábitos de vida saludable y el desarrollo de los valores de los valores como el espíritu de sana competición y juego limpio.

En el Capítulo XIII se instituye la formación en valores en el marco de la formación integral de las personas, y donde las Universidades promoverán actuaciones encomendadas al fomento de estos valores.

Las actividades de participación social y cooperación al desarrollo, se expresan en el Capítulo XIV, donde se pueden apreciar los compromisos de los estudiantes en relación a los derechos y deberes derivados de estas actividades.

La atención al estudiante universitario como herramienta complementaria a su formación integral, incluidos los servicios de alojamiento, se ha regulado en el Capítulo XV.

Finaliza el texto en el Capítulo XVI con una dedicación a las Asociaciones de Antiguos Alumnos. 